\title{
The prognostic value of chest X-ray in patients with COVID-19 on admission and when starting CPAP
}

\author{
Authors: Will Sargent, ${ }^{\mathrm{A}}$ Sajeed Ali, ${ }^{\mathrm{A}}$ Sebi Kukran, ${ }^{\mathrm{A}}$ Miranda Harvie ${ }^{\mathrm{B}}$ and Susanne Soin ${ }^{\mathrm{B}}$
}

\section{Objective}

The objective was to explore if chest X-ray severity, assessed using a validated scoring system, predicts patient outcome on admission and when starting continuous positive pressure ventilation (CPAP) for COVID-19.

Design

The study was a retrospective case-controlled study.

Participants

There were 163 patients with COVID-19 deemed candidates for CPAP on admission, including 58 who subsequently required CPAP.

\section{Outcome measures}

On admission, we measured the proportion of patients meeting a composite 'negative' outcome of requiring CPAP, intubation or dying versus successful ward-based care. For those escalated to CPAP, 'negative' outcomes were intubation or death versus successful de-escalation of respiratory support.

Results

Our results were stratified into tertiles, those with 'moderate' or 'severe' X-rays on admission had significantly higher odds of negative outcome versus 'mild' (odds ratio (OR) 2.32; $95 \%$ confidence interval (CI) 1.121-4.803; $p=0.023$; and OR 3.600; $95 \%$ CI 1.681-7.708; $p=0.001$, respectively). This could not be demonstrated in those commencing CPAP (OR 0.976; 95\% CI 0.754-1.264; $\mathrm{p}=0.856$ ).

\section{Conclusions}

We outline a scoring system to stratify X-rays by severity and directly link this to prognosis. However, we were unable to demonstrate this association in the patients commencing CPAP.

KEYWORDS: radiology, COVID-19, CPAP, chest X-ray, acute medicine

DOI: 10.7861/clinmed.2020-0576

\section{Introduction}

COVID-19, caused by the pandemic of SARS-CoV-2, encompasses a spectrum of clinical manifestations ranging from mild symptoms

Authors: Afoundation year doctor, Ealing Hospital, London, UK; ${ }^{B}$ consultant radiologist, Ealing Hospital, London, UK to severe type 1 respiratory failure necessitating ventilatory support. ${ }^{1}$

Although initially controversial, continuous positive airway pressure ventilation (CPAP) has been used routinely at our institution to improve oxygenation and reduce work of breathing in those with severe disease. ${ }^{2,3}$ So far, it is unclear what factors predict if a patient is likely to be 'weaned' from this oxygen delivery system and which will go on to require intubation or palliation if further escalation would not be appropriate. Given the limited number of CPAP machines and the intensive nursing regimens required, prognostication when starting therapy would enable more effective resource allocation in addition to highlighting those at risk of further deterioration. Similarly, identifying those likely to need CPAP on admission means those with severe disease who are at risk of deterioration can be initiated on therapy in a timely manner.

Numerous clinical and biochemical features have been shown to predict development of severe disease, including age, ethnicity, presence of specific comorbidities (such as hypertension and diabetes), raised inflammatory markers (such as C-reactive protein (CRP)), and lymphopenia manifesting in absolute terms and as a high neutrophil-to-lymphocyte ratio (NLR). ${ }^{4,5}$ To our knowledge, these have not been examined in relation to prognosis in patients on CPAP.

One further source of prognostic information could be the chest $X$-ray. Recent guidelines on chest X-ray reporting in COVID-19 released by the British Society of Thoracic Imaging (BSTI) recommend grading images into 'mild', 'moderate' and 'severe' but provide no framework upon which this assessment should be made. ${ }^{6}$ In addition, at present it remains unclear to what extent $\mathrm{X}$-ray features reflect severity of disease burden.

In the 2003/2004 SARS outbreak, a range of methodologies for quantifying chest $X$-ray severity were shown to correlate with prognosis. ${ }^{7-9}$ During the current COVID-19 outbreak, one chest $X$-ray scoring system applied in the emergency department has been shown to correlate with need for admission and intubation in young patients. ${ }^{10}$ To our knowledge, the prognostic value of the chest $\mathrm{X}$-ray in patients with COVID-19 requiring more intensive respiratory support has not been investigated. The extent of pulmonary oedema on chest $\mathrm{X}$-ray has been shown to correlate with more severe disease and worse clinical outcomes in patients with acute respiratory distress syndrome (ARDS). ${ }^{11}$ While the role of ARDS in COVID-19 pathophysiology remains controversial, a related semi-quantitative severity score based on extent of consolidation has previously been shown to predict mortality in non-COVID-19 intensive care unit patients with a range of pathologies. ${ }^{12,13}$ 
We hypothesised that chest X-ray severity assessed at admission and when starting CPAP would be an independent predictor of clinical outcome.

\section{Methods}

\section{Selection and description of participants}

The admission cohort consisted of a selection of patients admitted with reverse transcription polymerase chain reaction test (RT-PCR) confirmed COVID-19 between 15 March 2020 and 06 April 2020, in addition to all patients that went on to require CPAP. Exclusion criteria were those who were not deemed suitable for non-invasive ventilation (NIV) at admission. Patients were followed up until discharge, or until start of NIV or intubation.

The CPAP cohort consisted of all patients treated with CPAP for type 1 respiratory failure secondary to COVID-19 pneumonitis at our institution between 23 March 2020 and 17 April 2020. The only exclusion criterion was prior use of domiciliary NIV. The patients were followed up until discharge from hospital, death or intubation.

The presence of hypertension, diabetes, respiratory disease, cardiovascular (CV) disease and chronic kidney disease (CKD) was assessed by review of medical notes and corroborated with historical blood tests where necessary. For the admission cohort, insufficient data were available to reliably diagnose CKD so it was not analysed. Ethnicity data was obtained from electronic notes and patients were dichotomised as white or from a black, Asian and minority ethnic (BAME) background.

\section{Technical information}

\section{Defining cohort outcomes}

Outcomes in the admission group were dichotomised into wardbased management or requirement for CPAP, intubation or death. Outcomes in the CPAP cohort were dichotomised as 'success' (defined as weaning and subsequent discharge from hospital) and 'failure' (the requirement of intubation, death on CPAP or withdrawal due to futility).

Ceiling-of-care decisions were made by the admitting consultant, after discussion with the patient and next-of-kin as appropriate, and constantly re-evaluated by ward clinicians. No formal criteria were established for deciding when to start a patient on CPAP, and

Table 1. X-ray scoring methodology. Each quadrant score is summed to give the overall $X$-ray score out

\section{of 16}

Description

Quadrant score

Normal lung appearance 0

Hazy opacification of less than half the quadrant

Dense opacification of less than half the quadrant Hazy opacification of more than half the quadrant

Dense opacification of more than half the quadrant

the decision was made by a medical consultant or senior registrar on a case-by-case basis. Similarly, there were no formal criteria for intubation, and decision to intubate was made by agreement of the anaesthetic and medical teams. Withdrawal of CPAP was determined by consultant physicians in consultation with the patient and/or their next-of-kin as appropriate.

\section{Chest $X$-ray scoring and clinical data}

Severity of chest $X$-ray findings were assessed by the method described previously. ${ }^{13}$ In brief, the image was divided into quadrants by the spine and the level of the carina. Each quadrant was assessed for extent of 'hazy' or 'dense' opacification, where 'dense' was defined as sufficient to obscure the anterior rib margin. Each quadrant was scored as shown in Table 1.

The X-rays were scored by consensus of two consultant radiologists who were blinded to patient outcome. Examples of the scoring system applied to COVID-19 X-rays are given in Fig 1.

For the admission cohort, the X-ray taken during the initial clerking was scored and inflammatory markers were obtained from admission bloods. For the CPAP cohort, the last X-ray taken prior to commencement of CPAP was scored and inflammatory markers recorded from their most recent blood tests. If no blood results were available from within 48 hours of a patient starting CPAP, their inflammatory markers were excluded from the analysis. Follow-up continued until intubation, death or discharge in the CPAP group, and until start of CPAP, intubation, death or discharge in the admission group.

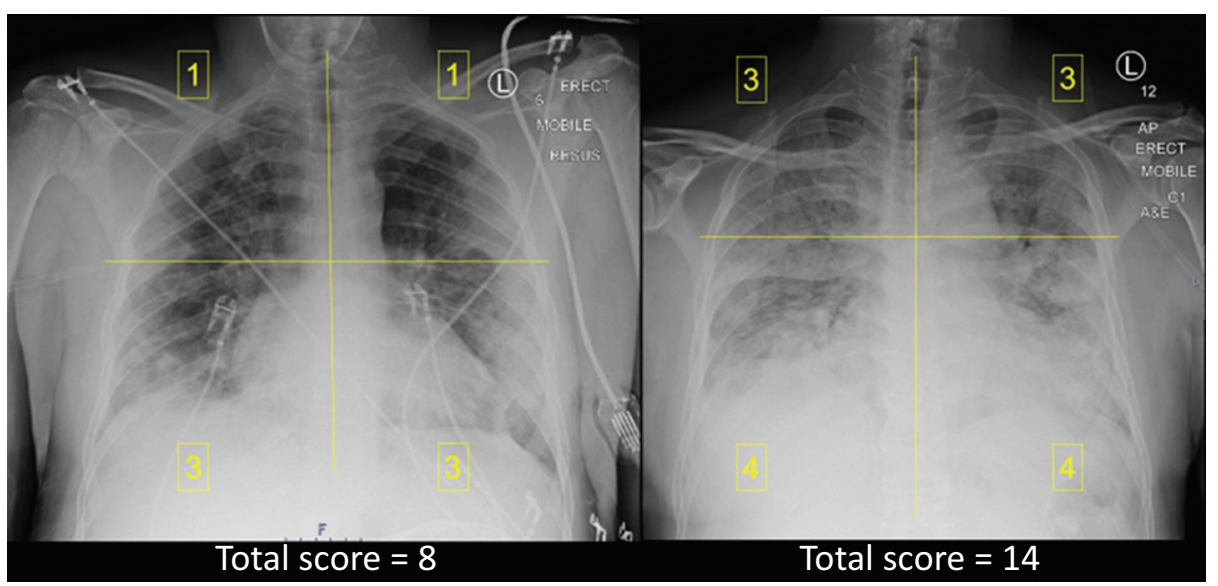

Fig 1. Two exemplum X-rays demonstrating the system of severity grading. 
Statistical analysis

Within the admission and CPAP cohorts, baseline characteristics between outcome groups were assessed for normality and compared by mean and independent samples t-test or median and Mood's median test, as appropriate. Prevalence of comorbidities between groups was presented as percentages and assessed by chi-squared test.

Predictive value of $X$-ray score on admission and starting CPAP was assessed by binomial logistic regression, with outcomes as previously defined. The analysis was adjusted for age, sex, inflammatory markers, presence of comorbidities and ethnicity. Goodness-of-fit was assessed by the Hosmer-Lemeshow test. $X$-rays of the admission and CPAP cohorts were separately ranked by severity score and stratified into tertiles post-hoc, and the regression analysis repeated with the same variables controlled. Time to outcome was visualised as Kaplan-Meier plots for each tertile, with significance assessed by the log-rank test applied pairwise between tertiles. For those discharged without event, censure date was set as three weeks after admission.

This work was approved by local ethics committee and carried out in accordance with institutional guidelines, approval reference SE20/037. All calculations were performed in IBM SPSS version 26.

\section{Results}

\section{Baseline characteristics}

\section{Admission cohort}

One-hundred and sixty-three patients were included, including all 58 patients who went on to require CPAP. Of the remaining 105 patients, 87 (82.9\%) were discharged after ward-based care and $18(17.1 \%)$ were intubated directly or died. A flow diagram of included patients and outcomes is given in Fig 2.

Demographic information and inflammatory markers on admission are given in Table 2 .

\section{CPAP cohort}

Fifty-eight patients were included, of which 23 (39.7\%) required intubation and 20 (34.5\%) died, leading to an overall CPAP success

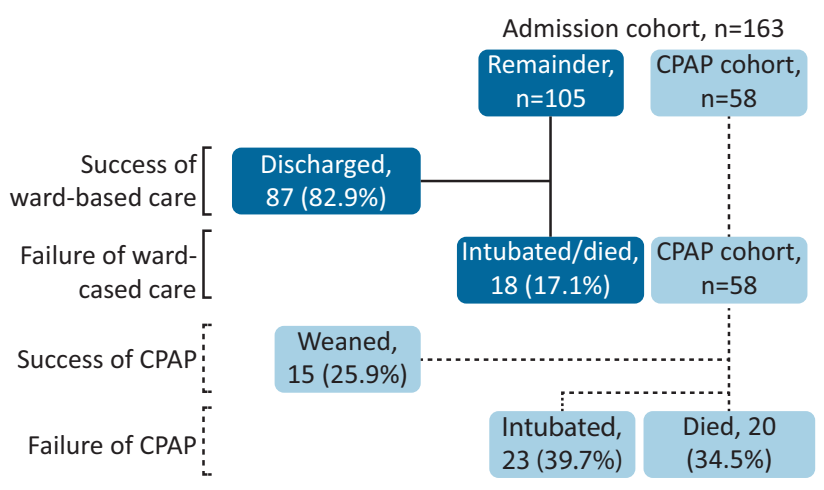

Fig 2. Outcome of included patients. CPAP = continuous positive airway pressure ventilation.

rate of $25.9 \%$. Patient characteristics and inflammatory markers on initiating CPAP are given below (Table 3).

\section{X-ray score prognostication}

\section{Admission cohort}

The median admission $\mathrm{X}$-ray severity score was significantly higher in the group that eventually went on to require respiratory support or die compared with those who did not (median score 9 vs 7; $\mathrm{p}=0.001)$

After controlling for age, sex, comorbidities, inflammatory markers and BAME status, the admission $\mathrm{X}$-ray severity score was a significant predictor of outcome with an OR of 1.274 (95\% CI 1.110-1.462; $p=0.001)$. Admission lymphocyte count was also significant with an OR of 0.315 (95\% CI $0.104-0.954 ; p=0.041)$. No other predictive variables reached significance in our analysis.

Patients were ranked by severity score and divided into tertiles corresponding to scores of $\leq 6,7-10$ and $\geq 11$ (mild, moderate and severe, respectively). Those with moderate and severe scores had significantly higher odds of requiring respiratory support or death

Table 2. Demographic information and admission inflammatory markers of the admission cohort

Total, $n=163$

56.2

78.5

75.4

38.9

32.7

16.7

Respiratory disease, \%

Cardiovascular disease, \%

Inflammatory markers

CRP (median), mg/L

Platelets (mean), $\times 10^{9} / \mathrm{L}$

NLR (median)

Absolute lymphocyte count (median), $\times 10^{9} / \mathrm{L}$
Uncomplicated, $\mathrm{n}=87$

CPAP/intubation/death $n=76$

$58.4^{\mathrm{a}}$

81.6

80.0

45.3

32.7

12.0

18.7

$163^{b}$

249

$7.5^{c}$

0.9

${ }^{a}=$ difference between mean by t-test $\mathrm{p}=0.044 ;{ }^{\mathrm{b}}=$ difference between median $\mathrm{p}<0.001 ;{ }^{\mathrm{c}}=$ difference between median $\mathrm{p}=0.012 ; \mathrm{BAME}=$ black, Asian and minority ethnic; CPAP = continuous positive airway pressure ventilation; CRP $=$ C-reactive protein; NLR $=$ neutrophil-lymphocyte ratio. 
Table 3. Demographic information and inflammatory markers when commencing continuous positive airway pressure ventilation

\begin{tabular}{|c|c|c|c|}
\hline & Total, $n=58$ & Weaned, $n=15$ & $\begin{array}{l}\text { Intubated/died, } \\
n=43\end{array}$ \\
\hline \multicolumn{4}{|l|}{ Demographic data } \\
\hline Age (mean), years & 59.2 & 52.6 & $61.6^{a}$ \\
\hline Male, \% & 76.3 & 93.3 & 72.1 \\
\hline BAME, \% & 79.2 & 76.9 & 80.0 \\
\hline Hypertension, \% & 51.7 & 46.7 & 53.5 \\
\hline Diabetes, \% & 37.9 & 26.7 & 41.9 \\
\hline Respiratory disease, \% & 17.2 & 6.7 & 20.9 \\
\hline Cardiovascular disease, \% & 22.4 & 13.3 & 25.6 \\
\hline Chronic kidney disease, \% & 25.9 & 13.3 & 30.2 \\
\hline \multicolumn{4}{|l|}{ Inflammatory markers } \\
\hline CRP (mean), mg/L & 239 & 261 & 230 \\
\hline Platelets (mean), $\times 10^{9} / \mathrm{L}$ & 284 & 294 & 280 \\
\hline NLR (median) & 8.5 & 8.2 & 8.7 \\
\hline Absolute lymphocyte count (median), $\times 10^{9} / \mathrm{L}$ & 1 & 0.9 & 1.0 \\
\hline
\end{tabular}

${ }^{a}=$ difference between mean $\mathrm{p}=0.009 ; \mathrm{BAME}=$ black, Asian and minority ethnic; $\mathrm{CPAP}=$ continuous positive airway pressure ventilation; $\mathrm{CRP}=\mathrm{C}$-reactive protein; $\mathrm{NLR}=$ neutrophil-lymphocyte ratio.

compared with mild (OR 2.32; 95\% CI 1.121-4.803; $p=0.023$, and OR 3.600; $95 \%$ CI 1.681-7.708; $p=0.001$, respectively). Difference in odds were also significantly different between each adjacent tertile (Fig 3a).

a

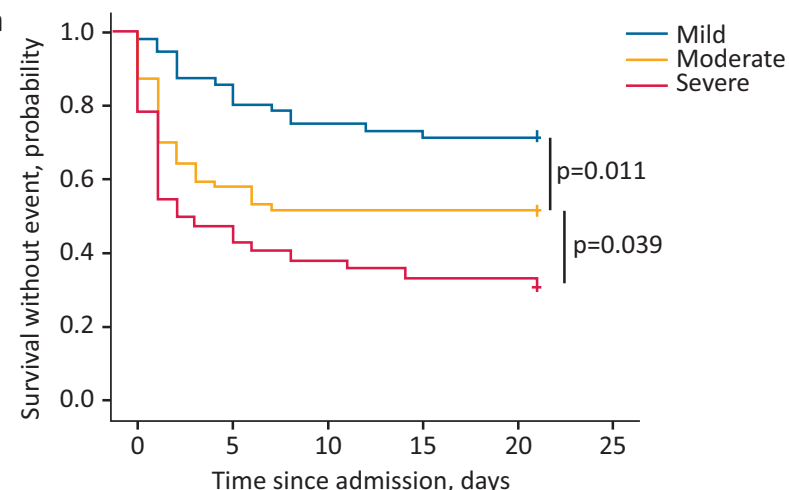

b

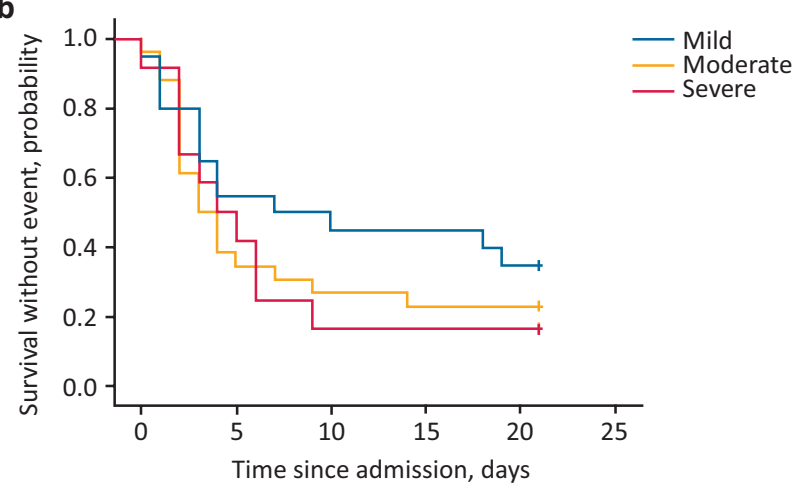

Fig 3. Time-to-event data of each chest X-ray severity tertile. a) The admission cohort. Black bars indicate significance as assessed by log-rank test. b) The continuous positive airway pressure ventilation cohort. There was no difference between tertiles as assessed by log-rank test.

\section{CPAP cohort}

The pre-CPAP $X$-ray scores were not significantly different between the outcome groups (wean vs intubation/death; 9 vs 11; $p=0.233$ ). After controlling for age, sex, inflammatory markers and BAME status, pre-CPAP X-ray severity was not a significant predictor of outcome in the logistic regression (OR 0.976; 95\% CI 0.754-1.264; $p=0.856)$. When grouped into equal tertiles by $X$-ray severity score $(0-8,9-12$ and $13+)$, there was no significant difference in timeto-outcome (Fig 3b).

\section{Association of $X$-ray severity with other biomarkers}

Admission chest $X$-ray score correlated moderately with admission CRP (Spearman's rho 0.495; $p<0.001$ ) and weakly with NLR (0.284; $p<0.001)$, but not with absolute lymphocyte or platelet count. There was no association between pre-CPAP X-ray score and inflammatory markers.

\section{Discussion}

In addition to playing an important role in diagnosing COVID-19, there is growing evidence that the chest $X$-ray may also play a role in prognostication. ${ }^{10}$ Given that chest $X$-ray is a ubiquitous and low-cost feature of initial patient assessment, any additional information gained from it has the potential to greatly impact routine patient care.

In COVID-19, it has become common practice to grade chest $\mathrm{X}$-rays as mild, moderate and severe based on subjective interpretation by the reviewer. However, it is unclear how these designations are arrived at and what relevance they have to patient management. Here, we have used a simple and validated scoring system to assign severity in a reproducible way. Stratifying patients on admission into tertiles by score boundaries of $\leq 6$, $7-10$ or $\geq 11$ produced three groups with significantly different risks of deterioration and death. We have demonstrated a system that can be used to define mild, moderate and severe $X$-rays and demonstrated a direct link between this and patient outcome; those in the moderate and severe groups have a 2.3- and 3.6-fold 
increase, respectively, in odds of requiring respiratory support or dying.

In our analysis, each 1-point increase in severity score increases the odds of requiring respiratory support or death by $27.4 \%$. This is broadly in keeping with the findings of other groups. ${ }^{10}$ However, to our knowledge, our study is the first to look at this association in a cohort that included patients older than 50 years. This is highly significant for two reasons. First, age is a significant predictor of severe COVID-19, so limiting the analysis to only the young risks neglecting a high-risk population. Secondly, although our study was limited to those physiologically robust enough to be candidates for CPAP, the average age of our population was almost 60 years, suggesting that exclusion of patients above 50 years old would severely limit generalisability.

The correlation between inflammatory markers and chest $X$-ray appearance has not been previously investigated for COVID-19. We found CRP, and to a lesser extent the NLR, to be positively associated with $\mathrm{X}$-ray severity score, suggesting that the degree of lung pathology correlates with the extent of the systemic inflammatory response. In addition, we found that X-ray severity correlated with outcome even after controlling for CRP and NLR, suggesting that the $X$-ray has prognostic benefit beyond that of simply reflecting the magnitude of the inflammatory cascade.

We were unable to show association between $\mathrm{X}$-ray severity and outcome in the CPAP cohort. There are several possible explanations for this. The overall number of patients treated with CPAP is small, with an even smaller number of successfully weaned patients, leading to the possibility of insufficient statistical power to reject the false null hypothesis. The proportion of patients who weaned was also small, further compounding this issue. For many patients, their pre-CPAP X-ray were taken several days prior to initiation of NIV, so there may have been substantial progression of $X$-ray change in that time. Another possible explanation is that all patients who require CPAP already have advanced lung disease, with subsequent similarity in severity scores, and outcome is thus influenced by other factors that affect physiological reserve such as age and comorbidity burden.

Differing from the original paper outlining the scoring method, all X-ray images were scored by two senior radiologists rather than intensive care or respiratory physicians. ${ }^{13}$ This has the benefit of improving scoring reliability, as many of the included images were of suboptimal quality, leading to the possibility of misinterpretation by less experienced physicians. Many of the $X$-rays were anterior-posterior (AP) or portable X-ray films, reflecting the unstable nature of the patients and hospital policy of minimising patient movement between departments. Underinspiration due to respiratory failure and high body mass index as a risk factor for severe disease further complicate image interpretation, especially in a disease that disproportionately affects the lower lobes. ${ }^{14}$

This scoring system offers a more objective and reproducible assessment than the subjective mild/moderate/severe grading that is currently more widely applied. However, there were limitations in the assessment as described in the original paper. 'Dense' only applying when the overlying rib was obscured was felt to be limiting, as significant consolidation away from the lung-rib interface would not qualify for the more severe score and there were many cases where this was challengeable. The lung could be extensively opacified, with loss of bronchovascular markings, but with rib still visualised, thus scored as 'hazy'. It was felt that 'consolidation' (opacified lung with air bronchograms) or 'atelectasis' (opacified lung with volume loss), or simply 'lung more opacified than could be called "hazy"' would be better qualifiers for the term 'dense'. Rigid interpretation of the original scoring system ensures reproducibility but may have led to systematic underestimation of X-ray severity.

\section{Strengths and limitations}

The most significant limitation to our study was the limited size and, in particular, the small number of CPAP patients we were able to include. Subsequent analyses, perhaps pooled from several institutions, may well report findings that we were too underpowered to show here.

We chose to limit the admission cohort to those whose ceilings of care included NIV, which reduced the number of patients that could be included. This allowed focus on patients where risk stratification could influence management decisions, as those requiring admission who were not candidates for CPAP or intubation would be managed in the same way regardless of disease severity.

No specific criteria were used to determine when a patient should be started on CPAP or intubated. While these decisions were made only by experienced clinicians, the lack of standardisation may have resulted in a greater variation in disease severity in these patient cohorts, further complicating analysis of prognosis.

Reflecting local demographics, over $75 \%$ of patients in both the admission and CPAP cohorts were from a BAME background. While this does limit generisability, those from a BAME background are known to be at increased risk of developing severe disease, so early identification of potential deterioration is especially crucial.

We were not able to include clinical observations (such as respiratory rate) or arterial blood gas results in this study. A composite score of heart rate, acidosis, consciousness level, oxygenation and respiratory rate has been shown to predict NIV failure in patients with chronic obstructive pulmonary disease exacerbations and an index using oxygen saturation, fraction of inspired oxygen and respiratory rate has been demonstrated to predict outcomes using high-flow nasal cannula therapy in pneumonia. ${ }^{15,16}$ While the underlying pathology and ventilation method is different in our study, these variables are important indicators of respiratory function and their prognostic benefit may carry across to COVID-19 patients on CPAP. Further work investigating correlation of X-ray severity with existing prognosticative scores such as SOFA and APACHE II may allow for an integrative score that could be a powerful tool in the decisionmaking process when managing COVID-19.

The chest $X$-ray scoring system employed here is a simple one but has been validated in a number of systemic and respiratory pathologies. ${ }^{13}$ Beyond serving as a source of prognostic information, systematic reporting of COVID-19 chest X-rays could facilitate easier comparisons across institutions for clinical and research purposes. The simplicity of the scoring methodology means that general physicians could be taught to use it, so that this additional prognostic information could be applied even when formal radiologist reporting is not available due to time or resource constraints.

\section{Conclusion}

Severity of the admission chest $\mathrm{X}$-ray is an independent risk factor for deterioration in COVID-19. To our knowledge, this 
is the first time this has been demonstrated in a cohort that included patients over 50 years old. We propose a reproducible way of stratifying $X$-rays into mild, moderate and severe, and demonstrated that these designations have direct impact on patient outcome. Admission X-ray severity correlates with markers of systemic inflammation, but severity score had prognostic value after controlling for these factors, implying benefit beyond that of simply reflecting the magnitude of the inflammatory cascade. Further work might see integration of systematically assessed $\mathrm{X}$-ray severity scoring into other COVID-19 risk stratification systems, and validating these initial findings in a larger patient cohort. We were unable to demonstrate this association in patients commencing CPAP, but this may have been limited by that advanced disease state and small patient numbers we were able to include in this analysis.

\section{Key points}

> At present, it is unclear how to stratify COVID-19 chest X-rays by severity and what relevance this would have to patient management and outcome.

$>$ We present a systematic scoring system for quantification of $\mathrm{X}$-ray disease burden and demonstrate that this correlates with odds of deterioration and death at admission.

$>$ To our knowledge, we are the first to demonstrate this in a representative patient population and present an evidencebased rationale for $\mathrm{X}$-ray severity scoring.

\section{References}

1 Huang C, Wang Y, Li X et al. Clinical features of patients infected with 2019 novel coronavirus in Wuhan, China. Lancet 2020;395: 497-506.

2 Arulkumaran N, Brealey D, Howell D, Singer M. Use of non-invasive ventilation for patients with COVID-19: a cause for concern? Lancet Respir Med 2020;8:e45.

3 McEnery T, Gough C, Costello RW. COVID-19: Respiratory support outside the intensive care unit. Lancet Respir Med 2020;8:538-9.

4 Zhou F, Yu T, Du R et al. Clinical course and risk factors for mortality of adult inpatients with COVID-19 in Wuhan, China: a retrospective cohort study. Lancet 2020;395:1054-62.
5 Liu Y, Du X, Chen ] et al. Neutrophil-to-lymphocyte ratio as an independent risk factor for mortality in hospitalized patients with COVID-19. J Infect 2020;81:e6-12.

6 British Society of Thoracic Imaging. Thoracic imaging in COVID-19 infection: Guidance for the reporting radiologist: Version 2. BSTI, 2020.

7 Chau TN, Lee PO, Choi KW et al. Value of initial chest X-rays for predicting clinical outcomes in patients with severe acute respiratory syndrome. Am J Med 2004;117:249-54.

8 Antonio GE, Wong KT, Tsui ELH et al. Chest X-ray scores as potential prognostic indicators in severe acute respiratory syndrome (SARS). AJR Am J Roentgenol 2005;184:734-41.

9 Hui DSC, Wong KT, Antonio GE et al. Severe acute respiratory syndrome: correlation between clinical outcome and radiologic features. Radiology 2004;233:579-85.

10 Toussie D, Voutsinas N, Finkelstein M et al. Clinical and chest X-ray features determine patient outcomes in young and middle age adults with COVID-19. Radiology 2020;297:E197-206.

11 Warren MA, Zhao Z, Koyama T et al. Severity scoring of lung oedema on the chest X-ray is associated with clinical outcomes in ARDS. Thorax 2018;73:840.

12 Gattinoni L, Chiumello D, Rossi S. COVID-19 pneumonia: ARDS or not? Critical Care 2020;24:154.

13 Mason SE, Dieffenbach PB, Englert JA et al. Semi-quantitative visual assessment of chest $X$-ray is associated with clinical outcomes in critically ill patients. Respiratory research 2019;20:218.

14 Wong HYF, Lam HYS, Fong AH-T et al. Frequency and distribution of chest X-ray findings in COVID-19 positive patients. Radiology 2020:296:E72-8.

15 Duan J, Wang S, Liu P et al. Early prediction of noninvasive ventilation failure in COPD patients: derivation, internal validation, and external validation of a simple risk score. Ann Intensive Care 2019;9:108.

16 Roca $\mathrm{O}$, Caralt B, Messika J et al. An index combining respiratory rate and oxygenation to predict outcome of nasal high-flow therapy. Am J Respir Crit Care Med 2019;199:1368-76.

Address for correspondence: Dr Will Sargent, Ealing Hospital, Uxbridge Road, Southall, London, Middlesex UB1 3HW, UK. Email: william.sargent@nhs.net

Twitter: @WillSargent3 